\title{
Apoptosis-associated tyrosine kinase 1 inhibits growth and migration and promotes apoptosis in melanoma
}

\author{
Shuang $\mathrm{Ma}^{1}$ and Brian P Rubin ${ }^{1,2}$
}

Apoptosis-associated tyrosine kinase 1 (AATK1) was initially identified as a protein that was dramatically overexpressed during growth arrest and apoptosis of 32Dcl myeloblastic leukemia cells. AATK is expressed in different regions of the brain and may have a role in normal nervous system development by its dual functions of enhancing apoptosis of mature granule cells and promoting terminal neuronal differentiation of developing neurons. However, its function in cancer has never been studied. Melanoma is a tumor composed of transformed cells within the melanocyte lineage deriving from the embryonic neural crest. It has been shown that developmental pathways in neural crest cells have a direct bearing on melanoma formation and human metastatic melanoma cells express a dedifferentiated phenotype. We found that the expression levels of AATK are lower in metastatic melanoma cell lines compared with primary melanoma cell lines and normal human melanocytes. We found that depletion of AATK mRNA in metastatic melanoma cell lines enhanced cell migration in cell line derived from metastatic melanomas. Overexpression of AATK inhibited cell proliferation, colony formation, and promoted apoptosis in melanoma cell lines derived from primary and metastatic melanomas. Signal transduction pathway analysis revealed that Src is involved in regulating AATK. Our results demonstrate for the first time that AATK inhibits cell proliferation, colony formation, and migration, and also promotes apoptosis in melanoma cells. Laboratory Investigation (2014) 94, 430-438; doi:10.1038/labinvest.2014.13; published online 3 March 2014

KEYWORDS: AATK; melanoma; metastasis; tumorigenesis

Apoptosis-associated tyrosine kinase 1 (AATK1), also called lemur kinase 1 , was originally identified as a protein that was dramatically upregulated during terminal differentiation and apoptosis of 32Dcl3, a non-tumorigenic mouse myeloid cell line. ${ }^{1}$ It is predominantly expressed at high levels in different regions of the brain, whereas at relatively low levels in other tissues including heart, lung, kidney, and skeletal muscle. ${ }^{1-4}$ AATK contains a tyrosine kinase domain at the $\mathrm{N}$-terminus and a proline-rich $\mathrm{SH} 3$-binding motif consisting of 16 PXXP motifs at the C-terminus. Two isoforms of AATK have been reported and referred to as AATKA and AATKB. AATKA encodes a cytoplasmic protein with an open reading frame of 1317 amino acids, whereas AATKB contains an additional transmembrane domain at the N-terminus, which adds 57 amino acids.,5 Functional differences between the two isoforms have never been addressed. Most functional studies of AATK have been focused on the nervous system. The expression of AATK is upregulated during neuronal differentiation of P19 embryonal carcinoma cells induced by retinoic acid. ${ }^{4}$ Overexpression of exogenous AATK promotes both neuronal differentiation of human neuroblastoma $\mathrm{SH}$ SY5Y cells, ${ }^{2}$ neurite extension of immature cerebellar granule cells, ${ }^{3}$ and apoptosis of cerebellar granule cells. ${ }^{4}$ These results indicate that AATK might have a role in nervous system development by its dual functions of promoting terminal neuronal differentiation of developing neurons and enhancing apoptosis of mature cerebellar granule cells.

Melanoma is a malignant neoplasm composed of cells with melanocytic differentiation deriving from the embryonic neural crest. It has been shown that developmental pathways in neural crest cells have a direct bearing on melanoma formation and human metastatic melanoma cells express a dedifferentiated phenotype. ${ }^{6,7}$ We examined protein levels of AATK in different human melanoma cell lines and found that AATK levels in metastatic melanoma cell lines including COLO829, MeWo, and WM164 were much lower compared

\footnotetext{
${ }^{1}$ Department of Molecular Genetics, Cleveland Clinic and Lerner Research Institute, Cleveland, OH, USA and ${ }^{2}$ Robert J. Tomsich Pathology and Laboratory Medicine Institute, Cleveland Clinic and Lerner Research Institute, Cleveland, $\mathrm{OH}, \mathrm{USA}$

Correspondence: Dr BP Rubin, MD, PhD, Department of Anatomic Pathology, Cleveland Clinic and Lerner Research Institute, L25, 9500 Euclid Avenue, Cleveland, OH 44195, USA.
}

E-mail: rubinb2@ccf.org

Received 30 October 2013; revised 8 January 2014; accepted 16 January 2014 


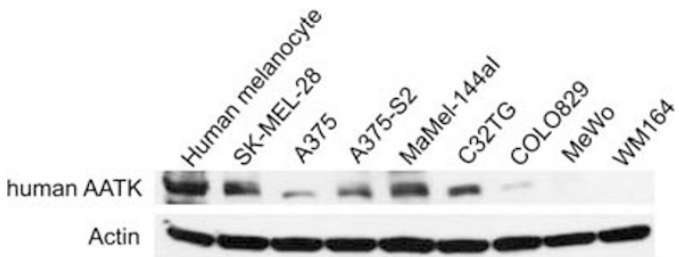

Figure 1 Differential expression levels of AATK in human melanoma cell lines. Cells were cultured in normal media, lysed, and cell lysates were immunoblotted with AATK antibody and actin. Data presented are representative blots from one of three separate experiments.

with primary melanoma cell lines including SK-MEL-28, A375, A375-S2, MaMel-144aI, and C32TG (Figure 1). Based on these findings, we hypothesized that AATK might have a role in melanoma tumorigenesis and metastasis. To test this hypothesis, we examined the influence of AATK expression on proliferation, apoptosis, anchorage-independent growth, and migration of human melanoma cell lines.

\section{MATERIALS AND METHODS}

\section{Expression Vectors and ShRNA vectors}

Full-length human AATKA-FLAG, human AATKB-FLAG, mouse AatkA-FLAG, and mouse AatkB-FLAG were inserted into the hygromycin-pBABE expression vector with FLAG at the $\mathrm{C}$ terminus. To obtain human AATKA-FLAG cDNA, multiple PCRs were performed using cDNA from COLO829 cells and primer sets 1,2 , and 3 (all primers are listed in Supplementary Table 1) and the PCR products were fused together by cold fusion reactions (System biosciences, Mountain View, CA, USA). To obtain human AATKB-FLAG cDNA, human AATKA was amplified by PCR using human AATKA-FLAG as template with primer set 4 . The additional $\mathrm{N}$-terminal fragment was amplified by PCR using COLO829 cDNA as template with primer set 5 . The two fragments were fused together by cold fusion reaction and inserted into hygro-pBABE. To obtain mouse AatkA-FLAG cDNA, PCR was performed using pYX-Asc-MGC Mouse Aatk cDNA (MMM1013-202799199, Thermal Scientific, Pittsburgh, PA, USA) as template with primer set 6 . To obtain mouse AatkBFLAG cDNA, mouse AatkA was amplified by PCR using mouse AatkA-FLAG construct as template with primer set 7 . An additional $\mathrm{N}$-terminal fragment was amplified by PCR using cDNA from Ink4a-Arf-deficient mouse melanocytes as a template with primer set 8 . The two fragments were fused together by a cold fusion reaction. All the primer sets are listed in Supplementary Table 1.

Human AATK shRNAs were also cloned into the pLKO.1puromycin lentiviral vector (Sigma-Aldrich, St Louis, MO, USA) and shRNA controls were purchased from SigmaAldrich (Supplementary Table 2).

\section{Antibodies}

Commercially available antibodies that were utilized include the following: mouse AATK (ab123363, Abcam, Cambridge,
MA, USA), human AATK (ab100857, Abcam); FLAG M2 (Sigma-Aldrich, St Louis, MO, USA); phospho-Src (Tyr416), Erk, Akt, phospho-Akt (Ser473), phospho-S6 (Ser235/236; Cell signaling, Beverly, MA, USA); c-Src, phospho-Erk (E-4), S6 (Santa Cruz Biotech, Santa Cruz, CA, USA).

\section{Cell Culture, Gene Transfer, and Gene Knockdown}

The following human melanoma cell lines were used in this study: SK-MEL-28, A375, A375-S2, MaMel-144aI, C32TG, COLO829, MeWo, and WM164. SK-MEL-28, A375, A375-S2, MaMel-144aI, and C32TG are from primary melanomas, whereas COLO829, MeWo, and WM164 are from metastatic melanomas. All of these cell lines contain BRAF V600E mutations with the exceptions of MaMel-144aI, which contains a KIT S476I mutation and MeWo, which does not contain BRAF, NRAS, KIT, or GNAQ mutations. Human melanoma cells including C32TG, MeWo, A375, A375-S2, SK-MEL-28, and WM164 were grown in DMEM supplemented with $10 \%$ fetal bovine serum (FBS), penicillin/ streptomycin, and MEM non-essential amino-acid solution (Sigma-Aldrich). COLO829 was grown in RPMI1640 supplemented with 10\% FBS, penicillin/streptomycin, and MEM non-essential amino-acid solution. MaMel-144aI was cultured in RPMI1640 supplemented with 10\% FBS and penicillin/streptomycin. Human melanocyte p'mel/hTERT/ CDK4(R24C)/p53DD was grown in Ham's F12 supplemented with $7 \%$ FBS, penicillin/streptomycin, $0.1 \mathrm{mM}$ IBMX, $50 \mathrm{ng} / \mathrm{ml} \mathrm{TPA}, 1 \mu \mathrm{M} \mathrm{Na} \mathrm{VO}_{4}$, and $1 \mu \mathrm{M}$ dbcAMP. All cell lines were maintained at $37^{\circ} \mathrm{C}$, in a humidified atmosphere of $5 \% \mathrm{CO}_{2}$.

Retrovirus was prepared by transfecting 293T cells with hygro-pBABE vector control or vector containing human AATKA-FLAG, human AATKB-FLAG, mouse AatkA-FLAG, or mouse AatkB-FLAG together with packaging plasmid pCL10A. C32TG, COLO829, and MeWo were infected by various retroviruses with $8 \mu \mathrm{g} / \mathrm{ml}$ polybrene for $8 \mathrm{~h}$ and then underwent hygromycin selection for 2 weeks starting $48 \mathrm{~h}$ after infection.

Lentivirus was prepared by transfecting 293T cells with pLKO.1-SHC1, SHC2, shRNA1, or shRNA2 together with VSVG and 48.2 . COLO829 cells were infected by various lentiviruses with $8 \mu \mathrm{g} / \mathrm{ml}$ polybrene for $8 \mathrm{~h}$ and then underwent puromycin selection for 10 days starting $48 \mathrm{~h}$ after infection.

\section{Cell Proliferation and Apoptosis Assays}

Three thousand cells were plated into 96-well clear plates for proliferation assays or five thousand cells were plated into white opaque plates for apoptosis assays. For cellular proliferation, cells were grown in complete media containing $10 \%$ FBS. Cellular proliferation was evaluated using Cell Counting Kit-8 (CCK8; Dojindo Laboratories, Kumamoto, Japan), according to the manufacturer's instructions. Apoptosis was evaluated $24 \mathrm{~h}$ after changing the media with phosphate-buffered saline by measuring caspase-3/7 activity using the Caspase Glo-3/7 assay system (Promega, Madison, WI, USA); luminescence was measured using a Wallac 1420 
multilabel counter (Perkin Elmer, Waltham, MA, USA). For all experiments, cells were plated in at least six wells and each experiment was performed three times.

\section{Soft Agar Colony Formation and Cell Migration}

The bottom agar was built from solidification of $2 \mathrm{ml}$ complete media containing $0.5 \%$ agar in a six-well flat-bottomed plate. Cell suspensions containing 10000 cells in $0.35 \%$ agar in complete media were poured to form the top layer. After the top layer solidified at room temperature for $3 \mathrm{~h}$, colonies were allowed to grow for 28 days at $37^{\circ} \mathrm{C}$ with $5 \% \mathrm{CO}_{2}$ before imaging.

A Boyden chamber assay was used to evaluate cell migration. A total of 40000 C32TG cells or 200000 COLO829 or MeWo cells in serum-free medium containing $10 \mu \mathrm{g} / \mathrm{ml} \mathrm{BSA}$ was plated onto the porous membrane $(8.0-\mu \mathrm{m}$ pore size) of the upper chamber of Transwell migration chambers (Millipore). Cell migration was allowed to proceed at $37^{\circ} \mathrm{C}$ for $22 \mathrm{~h}$ and then cells that migrated to the underside of membrane were stained with DAPI and counted. For each membrane, five medium-power fields were counted and all assays were performed in triplicate.

\section{Immunoblotting and Immunoprecipitation}

Cells were grown to sub-confluency, collected, lysed in radioimmunoprecipition assay buffer (50 mM Tris PH 8.0, 1\% NP-40, 0.25\% sodium deoxycholate, $150 \mathrm{mM} \mathrm{NaCl}$ ) plus Mini EDTA-free Protease Inhibitor Cocktail and PhosSTOP (Roche Applied Science, Indianapolis, IN, USA) and extracts were obtained by centrifugation at $10000 \mathrm{~g}$ at $4{ }^{\circ} \mathrm{C}$. Protein lysates were subjected to $4-15 \%$ SDS-PAGE and immunoblotted with the corresponding primary antibody, followed by incubation with horseradish peroxidase-conjugated secondary antibody (Invitrogen, Carlsbad, CA, USA) and developed using Supersignal-ECL kit (Thermo Scientific-Pierce, Rockford, IL, USA) on a Bio-Rad ChemiDoc XRS imaging station. For immunoprecipitation, samples were incubated with $2 \mu \mathrm{g}$ antiFLAG M2 (Sigma-Aldrich) or anti-c-Src (Santa Cruz Biotech) antibodies overnight and immunoprecipitated by protein $\mathrm{G}$ plus Sepharose (Santa Cruz Biotech) for $2 \mathrm{~h}$ at $4{ }^{\circ} \mathrm{C}$. The immune complexes were washed three times with lysis buffer. Proteins were separated by SDS-PAGE.

\section{Statistics}

Each experiment was repeated three times. Differences in measured variables between experimental and control groups were assessed by using one-way analysis of variance followed by Dunnet's multiple comparison test using Prism 5 software (GraphPad, La Jolla, CA, USA). Statistical significance was accepted at $P<0.05$.

\section{RESULTS}

\section{Differential Expression Levels of AATK in Melanoma Cell} Lines

To determine the level of AATK expression in the human melanocyte p'mel/hTERT/CDK4(R24C)/p53DD cell line, five primary melanoma cell lines, including SK-MEL-28, A375, A375S2, MaMel144aI, and C32TG and three metastatic melanoma cell lines, including COLO829, MeWo, and WM164, cell lysates were immunoblotted with antibodies that recognize the C-terminus of human AATK. AATK was expressed at relatively high levels in human melanocytes and most primary melanoma cell lines, whereas it was barely expressed or expressed at very low levels in metastatic melanoma cell lines (Figure 1).

\section{Overexpression of AATK Inhibits Cell Proliferation, Anchorage-Independent Growth, and Promotes Apoptosis of Melanoma Cells.}

To explore the functions of AATK in melanoma cells, expression vectors containing mouse AatkA-FLAG, mouse AatkB-FLAG, human AATKA-FLAG, or human AATKBFLAG were introduced into the C32TG primary melanoma cell line and COLO829 and MeWo metastatic melanoma cell lines and proliferation, colony formation in soft agar (ability to grow in an anchorage-independent manner), apoptosis, and cell migration were evaluated. All three cell lines with AATK expression exhibited a modest decrease in proliferation when they were cultured in complete media in comparison with vector only controls (Figure 2a). The ability to form colonies in soft agar was also inhibited in all three melanoma cell lines by the introduction of exogenous AATK (Figure 2c). When cell apoptosis was measured, no obvious effect of AATK overexpression was observed when these melanoma cells were grown in complete media, or under serum starvation conditions including 2.5\% FBS and 0\% FBS for up to a week in culture (data not shown). However, when cells were cultured for a day in phosphate-buffered saline, a total serum-nutrient-glucose starvation condition, AATK expression dramatically enhanced cell apoptosis in all three melanoma lines (Figure 2b).

To clarify if the transformed phenotypes were caused by the introduction of exogenous AATK, overexpressed AATK was depleted by AATK shRNAs in COLO829 cells. Depletion of $A A T K$ by AATK shRNAs restored the changes in cell proliferation, anchorage-independent growth, and apoptosis in COLO829 cells with AATK overexpression (Figures $3 \mathrm{a}, \mathrm{b}$ and $\mathrm{c}$ ), which confirmed that overexpression of AATK resulted in inhibition of cell proliferation, anchorageindependent growth, and promotion of apoptosis in melanoma cells.

\section{Depletion of AATK Promotes Migration of Melanoma Cells}

Depletion of AATK by AATK shRNA2 in AATK-overexpressing COLO829 cells dramatically increased cell migration, whereas $A A T K$ shRNA1 did not affect migration (Figure $3 \mathrm{~d}$ ). Western blot analysis revealed that AATK shRNA1 diminished AATK expression to a level similar to the level in control cells, whereas AATK shRNA2 almost abolished AATK expression completely (Figure 3e). Conversely, 
depletion of AATK by shRNAs in COLO829 and C32TG cells revealed that both AATK shRNA1 and shRNA2 decreased the expression level of AATK as shown by western blot analysis (Figures $4 \mathrm{c}$ and $\mathrm{d}$ ) and both shRNAs significantly increased cell migration in AATK knockdown cells compared with controls (Figures $4 \mathrm{a}$ and $\mathrm{b}$ ).

\section{Inverse Correlation Between Expression Level of AATK} and Activation Level of Src in COLO829 and MeWo Cells

We next investigated the signal transduction pathways that mediate AATK's functions. We examined Src as a potential candidate because a physical association between AATK and Src was reported in mouse brain microsome fractions and in
COS-7 cells. ${ }^{8}$ We detected total protein levels and activation levels of Src in COLO829 and MeWo cells by western blot analysis. Our results showed that there was a dramatic decrease of pY416 Src, the active form of Src, in COLO829 and MeWo cells with AATK overexpression compared with control cells with no change in total Src protein levels (Figure 5). In addition, when the overexpression of human AATKA in COLO829 was depleted by shRNAs, pY416 Src levels went up correspondingly (Figure 3e). Knocking down AATK in naive COLO829 cells also led to a similar result (Figure 4d). All these results demonstrated that an inverse correlation between the activation of Src and expression of AATK in COLO829 and MeWo cells.
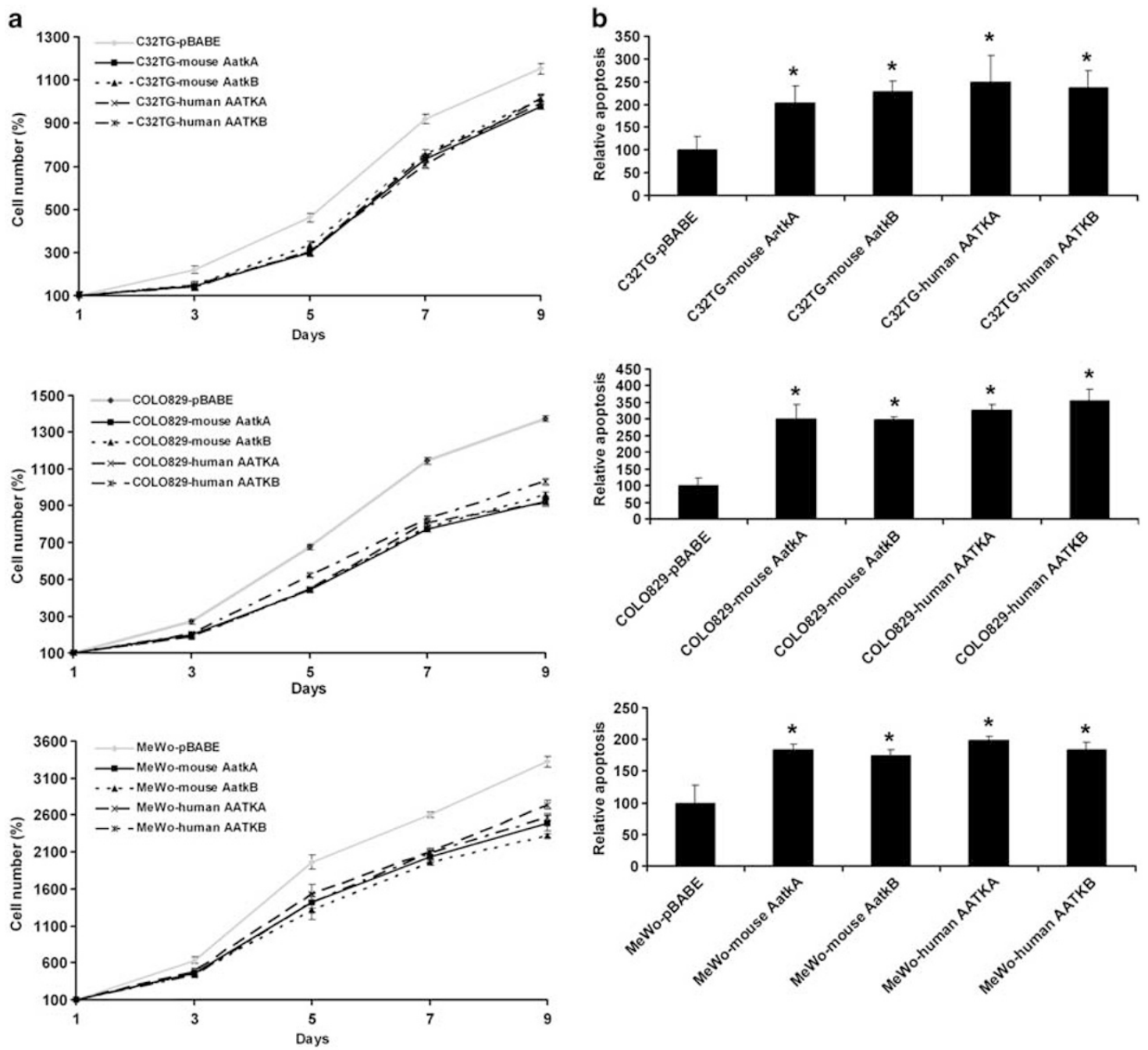

Figure 2 Overexpression of AATK inhibits cell proliferation and colony formation, and enhances apoptosis in C32TG, COLO829, and MeWo melanoma cells. C32TG, COLO829, and MeWo cells expressing pBABE vector control alone, mouse AatkA-FLAG, mouse AatkB-FLAG, human AATKA-FLAG or human $A A T K B$-FLAG were used in the following experiments. (a) Cell proliferation was measured in cells cultured in normal media at different time points with the data normalized to the corresponding baseline at 1 day. (b) Cells were grown for $24 \mathrm{~h}$ in PBS and assayed for caspase 3/7 activity with the data normalized to the baseline cell numbers and compared with the corresponding controls. ${ }^{*} P<0.05$. (c) Representative images and quantitation from soft agar colony formation assay for cells expressing AATK compared with the corresponding controls. All values are expressed as means \pm s.d. Each experiment was performed three times. ${ }^{*}=P<0.05$. 
C
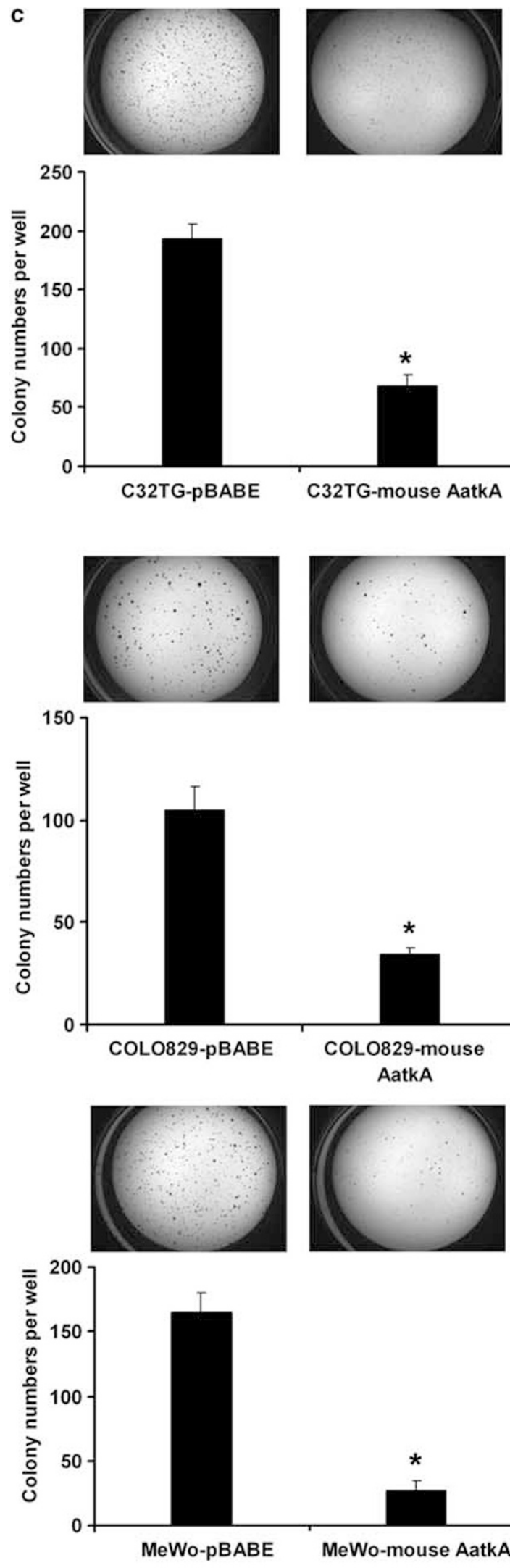

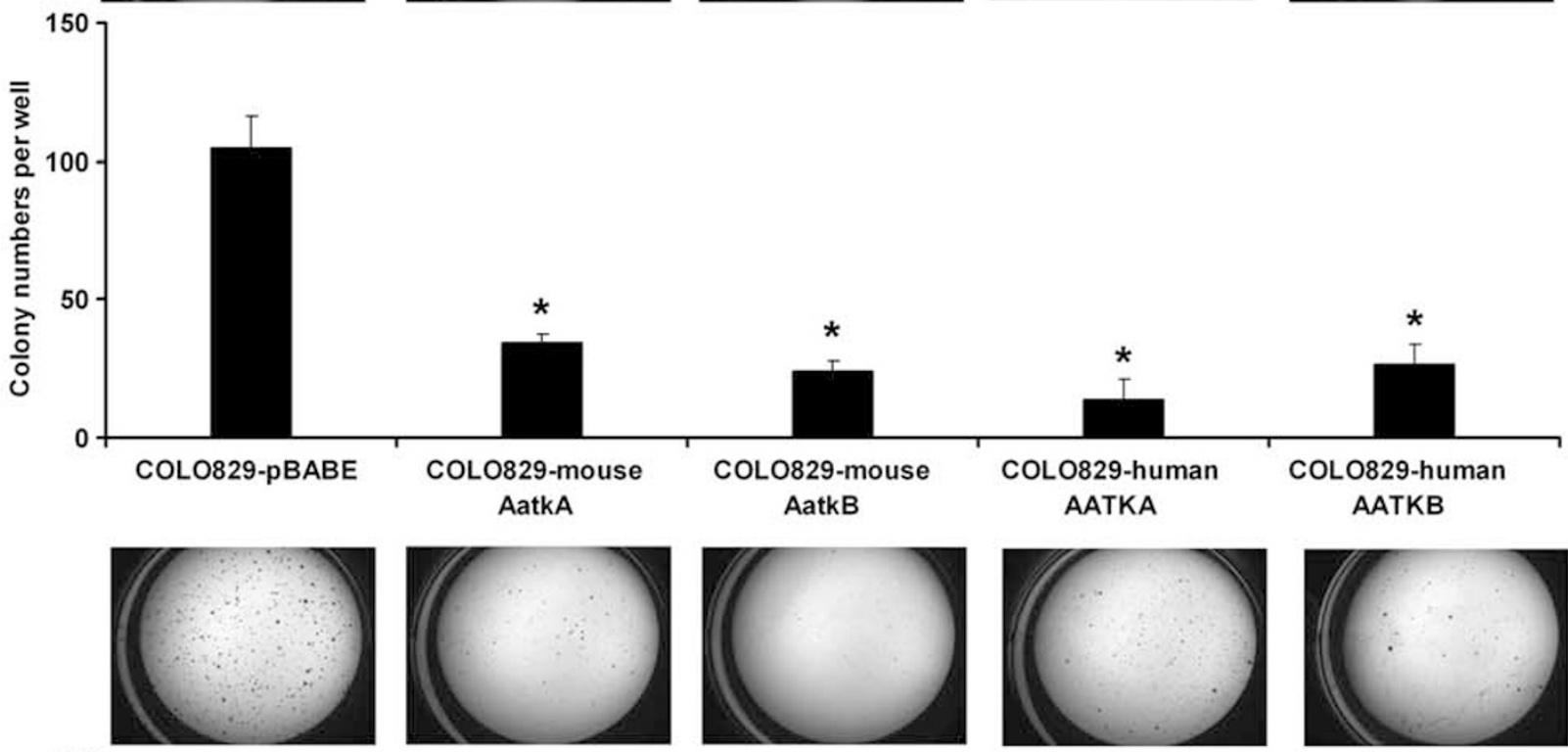

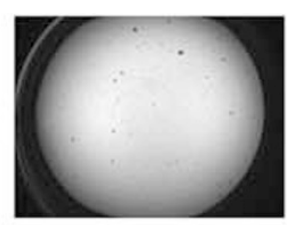
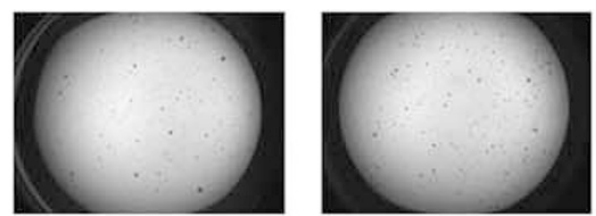
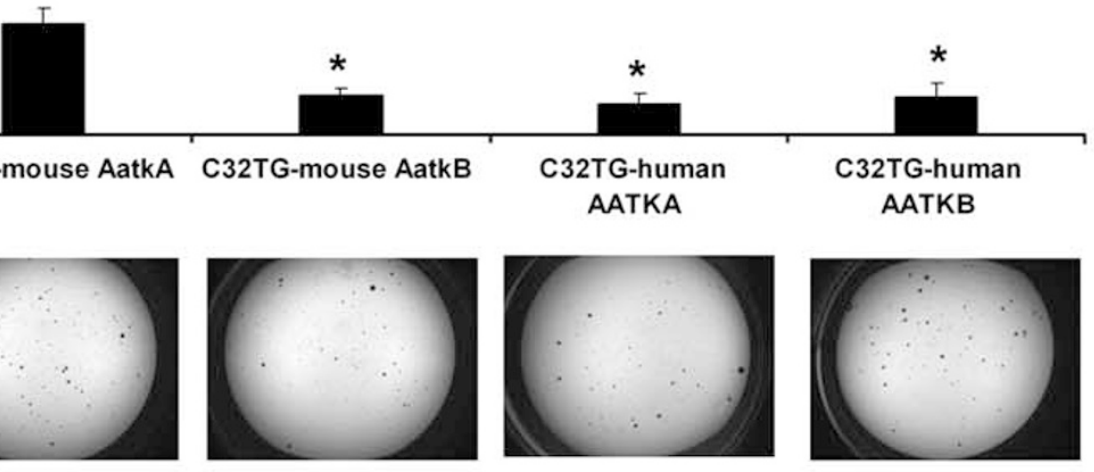

32TG-human

AATKB
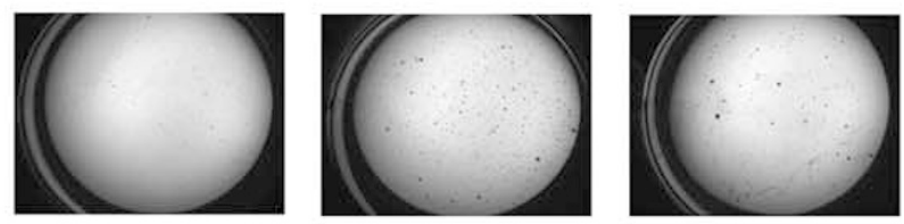

Figure 2 Continued. 
a

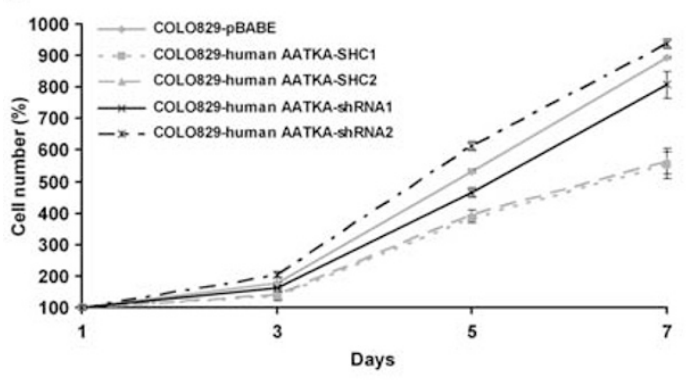

b

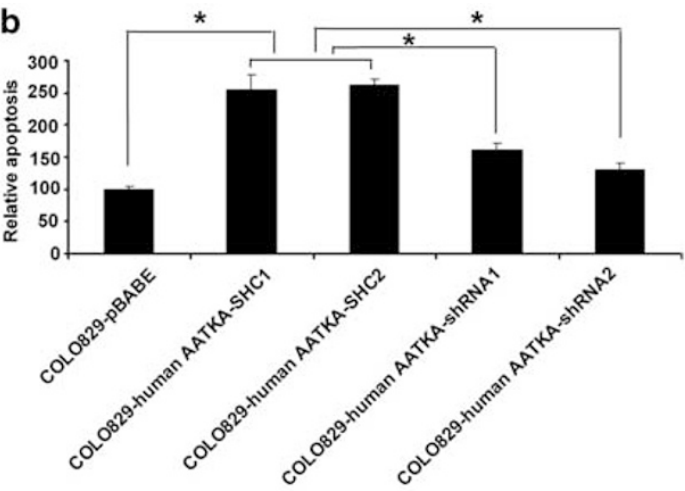

C
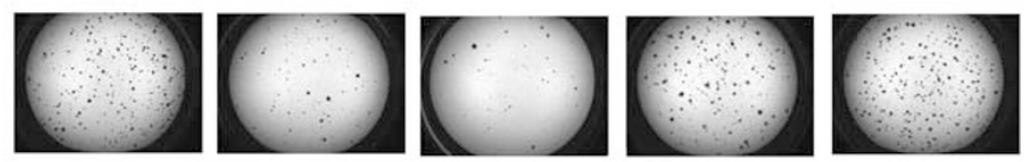

*

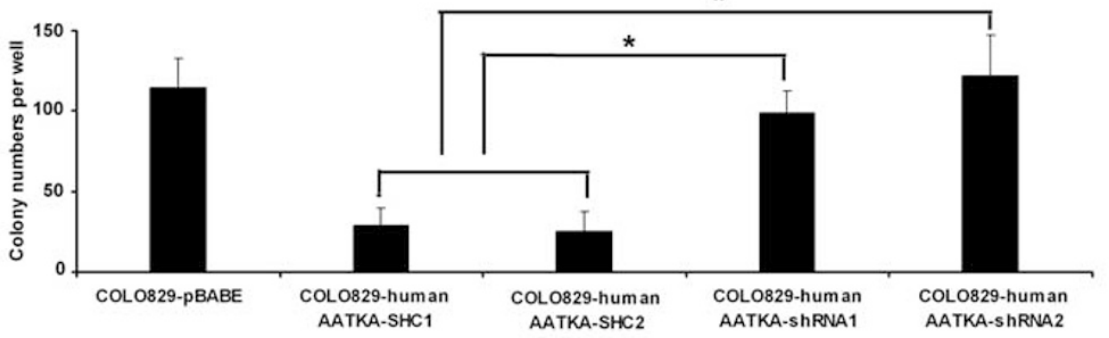

e

d
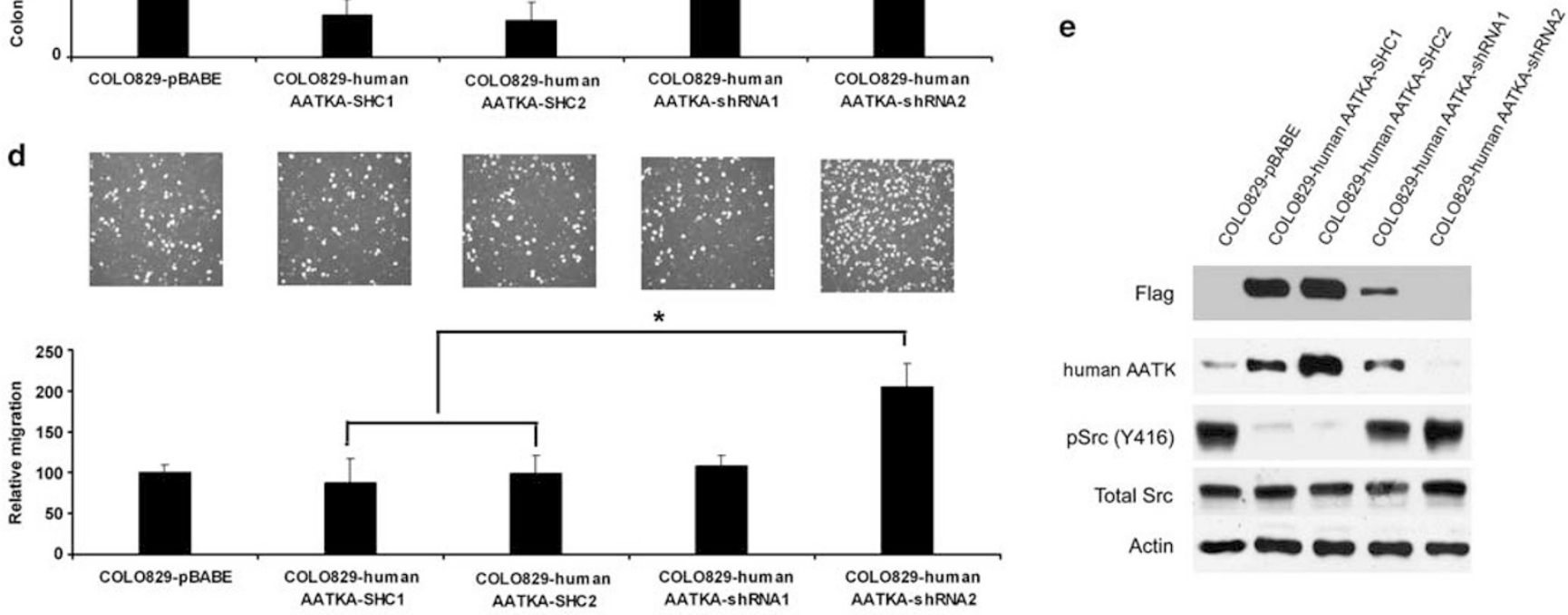

Figure 3 Knockdown of AATK restores cell proliferation, colony formation, apoptosis, and increases cell migration in COLO829-hAATKA melanoma cells (COLO829 cells expressing human AATKA-FLAG). COLO829-hAATKA cells transfected with SHC1 (pLKO.1 empty vector control), SHC2 (pLKO.1 with nonmammalian shRNA control), shRNA1 (pLKO.1 AATK shRNA1), or shRNA2 (pLKO.1 AATK shRNA2) and COLO829 cells with pBABE vector only were used in the following experiments. (a) Cell proliferation was measured in cells cultured in normal media at different time points with the data normalized to the corresponding baseline at 1 day. (b) Cells were grown for $24 \mathrm{~h}$ in PBS and assayed for caspase 3/7 activity with the data normalized to COLO829pBABE. ${ }^{*} P<0.05$. (c) Representative images and quantitation from soft agar colony formation assay. ${ }^{*} P<0.05$. (d) All values are expressed as means \pm s.d. (a-d) Representative images and quantitation from Boyden chamber migration assay performed $24 \mathrm{~h}$ after seeding cells with data normalized to COLO829-pBABE. ${ }^{*} P<0.05$. (e) Cell lines were cultured in normal media, lysed, and cell lysates were immunoblotted with antibodies for human AATK, pSrc (Y416), Src, and actin. Data presented are representative blots from one of three separate experiments. Each experiment (a-e) was performed three times.

To interrogate the interaction between Src and AATK in these melanoma cells, we examined whether Src could be co-immunoprecipitated with FLAG-AATK in MeWo cells with FLAG-AATK overexpression and naïve MeWo cells. Src was not detected in the immunoprecipitation with anti-FLAG or human anti-AATK, nor FLAG or AATK was detected in the immunoprecipitation with anti-Src (data not shown), indicating no visible direct interaction between Src and AATK.

To further investigate the signaling pathways contributing to AATK's functions, the PI3K pathway and mitogen-activated protein kinase (MAPK) pathway were examined. No differences in phosphorylation of AKT or S6, two readouts of 
a
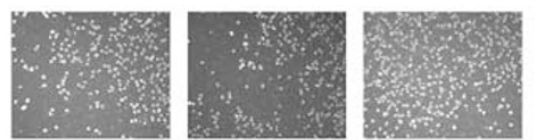

*

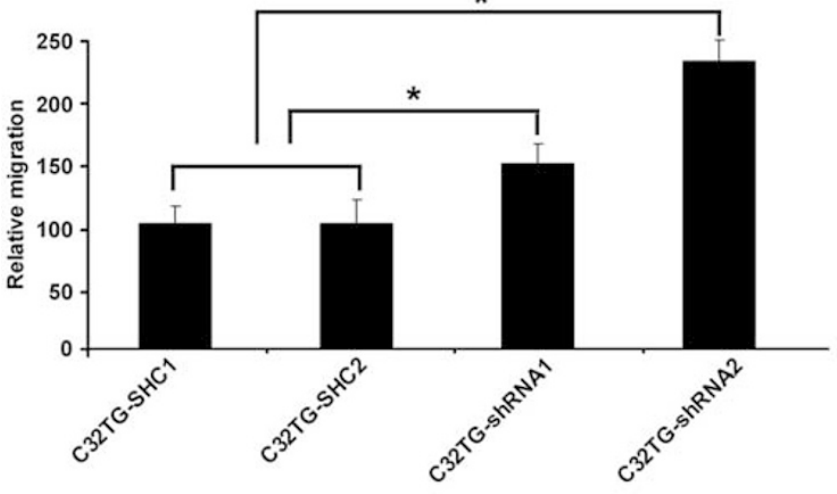

C

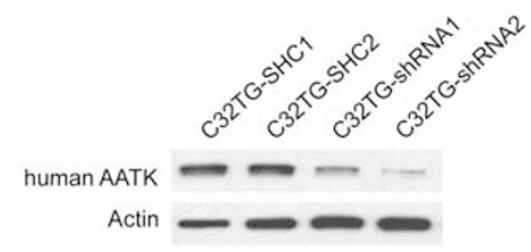

b
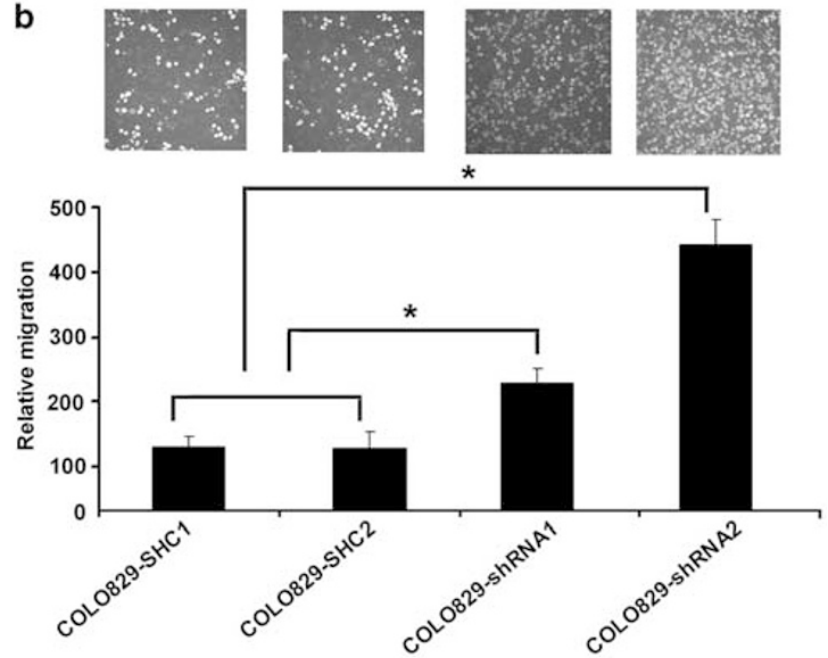

d

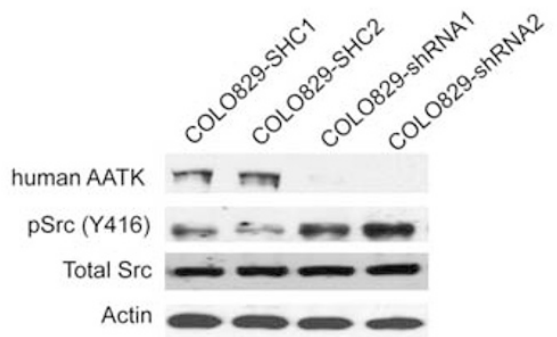

Figure 4 Knockdown of AATK in naive C32TG and COLO829 melanoma cell lines enhances cell migration. C32TG or COLO829 cells transfected with either SHC1 (pLKO.1 empty vector control), SHC2 (pLKO.1 with non-mammalian shRNA control), shRNA1 (pLKO.1 AATK shRNA1) or shRNA2 (pLKO.1 AATK shRNA2) were used in the following experiments. $(\mathbf{a}, \mathbf{b})$ Representative images and quantitation from Boyden chamber migration assay performed $24 \mathrm{~h}$ after seeding cells with data normalized to either C32TG-SHC1 or COLO829-SHC1 correspondingly. All values are expressed as means \pm s.d. ${ }^{*} P<0.05$. (c, d) Cells were cultured in normal media, lysed, and lysates were immunoblotted with antibodies for human AATK and actin. Data presented is a representative blot from one of three separate experiments. Each experiment (a-d) was performed three times.

activation of the PI3K pathway, nor in phosphorylation of ERK, a readout of the MAPK pathway were observed (Figure 5).

\section{DISCUSSION}

AATK has been studied primarily in the nervous system. No studies have examined the function of AATK in cancer. Melanoma cells originate from the neural crest, which is the same as neurons. Melanoma pathogenesis has been compared with neural crest cell development. AATK is known to be involved in the development of the nervous system. Although AATK regulates neuronal differentiation, so-called 'dedifferentiation' is observed in metastatic melanoma. Although AATK is expressed at very low, almost undetectable levels in other tissues except for brain as reported, ${ }^{9}$ it is detected at a relatively high level in human melanocytes and a moderate level in several melanoma cell lines derived from primary (non-metastatic) melanomas (Figure 1). The downregulation of AATK expression observed in cell lines derived from metastatic melanomas compared with cell lines derived from primary melanomas and melanocytes suggested a possible role in tumor progression of melanoma. Therefore, we investigated the functions of AATK in tumorigenesis and progression using melanoma cell lines as a model system.

We observed that two major isoforms of AATK, AATKA and AATKB, inhibited cell proliferation, colony formation, and cell migration and promoted cell apoptosis in melanoma cells. In human melanocytes, the relatively high expression levels of AATK likely help maintain the normal rate of cellular proliferation and apoptosis. In cell lines from primary melanomas, low to moderate expression levels of AATK do not affect tumor cell proliferation but they do inhibit cellular mobility. The expression of AATK in metastatic melanoma cell lines was downregulated to almost undetectable levels, suggesting that AATK may indeed have a role in metastasis. Because AATK promotes apoptosis, it could exert an anti-metastatic effect either through promotion of anoikis or apoptosis during attempts at colonization of distant organs.

The pro-apoptotic functions and anti-proliferative functions of AATK observed in melanoma cells are independent of each other. Exogenous AATK inhibits melanoma cellular proliferation in complete media without affecting apoptosis 


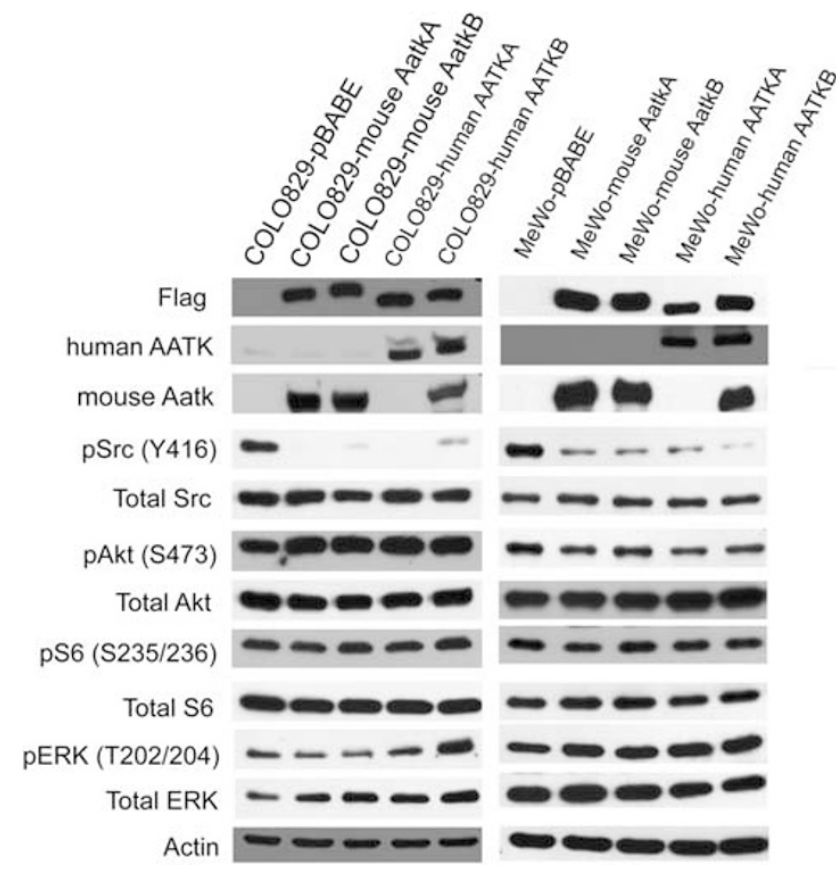

Figure 5 Inverse correlation between expression level of AATK and activation level of Src in COLO829 and Mewo melanoma cells. COLO829 and MeWo cells expressing pBABE vector control alone, mouse AatkAFLAG, mouse AatkB-FLAG, human AATKA-FLAG, or human AATKB-FLAG were cultured in normal media, and lysates were immunoblotted with antibodies for FLAG, human AATK, mouse AATK, pSrc (Y416), Src, pAkt (S473), Akt, pS6 (S235/236), S6, pERK (T202/204), ERK, and actin. Data presented are representative blots from one of three separate experiments.

(data not shown), whereas the pro-apoptotic function of AATK is only observed in the apoptosis induced by depriving cells of growth factors and nutrients, including amino acids, glucose, and potassium chloride. This pro-apoptotic function of AATK is similar to what has been observed in myeloid precursor cells ${ }^{1}$ and cerebellar granule cells. ${ }^{9}$

A previous study has shown a physical interaction between AATK and Src in mouse brain microsome fractions and COS 7 cells. ${ }^{8}$ Src is a member of the Src family kinases), which regulate multiple biological processes including cell proliferation, survival, adhesion, and migration, depending on the cellular context. ${ }^{10-12}$ SRC also contributes to cancer development. ${ }^{13-15}$ SRC is activated in various kinds of cancers including breast cancer, colorectal cancer, and melanoma. ${ }^{16,17}$ The different levels of SRC activation in primary and metastatic melanomas suggest involvement of SRC in the progression of melanoma, ${ }^{18}$ which is consistent with the correlation between increases in SRC kinase activity and the progression of malignancy observed in colon cancer. ${ }^{19}$ Other data also support an important role of SRC in melanoma cell growth and migration. ${ }^{20-23}$ According to these observations, we proposed that SRC might mediate AATK functions in melanoma cells. Therefore, we examined total protein levels and SRC activation levels in COLO829 and MeWo cells by western blot analysis. Activation of Src depends on the phosphorylation of two crucial tyrosine residues, Y416 and Y530. In the inactive state, Y530 is phosphorylated and binds to its $\mathrm{SH} 2$ domain to promote a closed conformation preventing the interaction with substrates through the association between the $\mathrm{SH} 3$ domain and the linker domain on the backside of the catalytic domain. When Y530 is dephosphorylated, the SH2 and SH3 domains are released from the intramolecular interactions and the open conformation allows the phosphorylation of Y419 in the activation loop to enhance the catalytic activity. ${ }^{24}$ Activation of Src correlates with Y419 levels. Our results revealed an inverse correlation between the activation of SRC and expression of AATK in COLO829 and MeWo cells, which implicates SRC in AATK function in melanoma cells. Our immunocytochemical analysis also showed a punctate perinuclear accumulation of AATK in melanoma cells (Supplementary Figure 1), consistent with localization of AATKA in recycling endosomes of COS-7 cells. ${ }^{8}$ As it has been known that the intracellular location of SRC is in the endosome, especially the late endosome, ${ }^{25}$ we wondered whether AATK might directly regulate the activity of SRC in melanoma cells. However, when we looked for a physical association between SRC and AATK in MeWo cells by immunoprecipitation, we were unable to detect an association between them. One possibility is that the interaction between AATK and Src is dependent on the cellular context. In MeWo cells, AATK may regulate the activity of SRC indirectly through intermediate factors so it would not directly interact with SRC itself. Another possibility is that the active state of SRC is crucial for its binding. As SRC phosphorylation was dramatically decreased in MeWo cells with AATK overexpression, we were not able to see the binding between exogenous AATK and SRC, whereas in naïve MeWo cells, AATK expression is too low to demonstrate an interaction.

We also further investigated the PI3K pathway and MAPK pathways, which can be activated by SRC and are crucial for cell survival, proliferation, and migration. ${ }^{24,26-28}$ However, we did not observe that SRC influenced either PI3K or MAPK signaling. As Src family kinases are key regulators of many signal transduction pathways, ${ }^{29}$ it is not surprising to find that SRC did not activate these two pathways in melanoma cells. Signaling pathways involved in SRC regulation, such as JAK-STAT and JNK-JUN pathways, should be explored in future studies to determine SRC's downstream effectors that mediate AATK function.

In conclusion, our study for the first time demonstrates that AATK inhibits cell proliferation, anchorage-independent growth, and cell migration and promotes apoptosis of melanoma cells. Therefore, AATK could be an important contributor to melanoma development and progression. The inverse correlation between expression level of AATK and activation level of SRC implicates SRC as an important mediator of AATK function in melanoma. 
Supplementary Information accompanies the paper on the Laboratory Investigation website (http://www.laboratoryinvestigation.org)

\section{ACKNOWLEDGMENTS}

This project was funded by a grant from the Melanoma Research Alliance.

\section{DISCLOSURE/CONFLICT OF INTEREST}

The authors declare no conflict of interest.

1. Gaozza E, Baker SJ, Vora RK, et al. AATYK: a novel tyrosine kinase induced during growth arrest and apoptosis of myeloid cells. Oncogene 1997;15:3127-3135.

2. Raghunath $M$, Patti $R$, Bannerman $P$, et al. A novel kinase, AATYK induces and promotes neuronal differentiation in a human neuroblastoma (SH-SY5Y) cell line. Brain Res Mol Brain Res 2000;77: 151-162.

3. Tomomura M, Hasegawa Y, Hashikawa T, et al. Differential expression and function of apoptosis-associated tyrosine kinase (AATYK) in the developing mouse brain. Brain Res Mol Brain Res 2003;112: 103-112.

4. Tomomura M, Morita N, Yoshikawa F, et al. Structural and functional analysis of the apoptosis-associated tyrosine kinase (AATYK) family. Neuroscience 2007;148:510-521.

5. Baker SJ, Sumerson R, Reddy CD, et al. Characterization of an alternatively spliced AATYK mRNA: Expression pattern of AATYK in the brain and neuronal cells. Oncogene 2001;20:1015-1021.

6. White RM, Cech J, Ratanasirintrawoot S, et al. DHODH modulates transcriptional elongation in the neural crest and melanoma. Nature 2011;471:518-522.

7. Hendrix MJ, Seftor EA, Hess $A R$, et al. Vasculogenic mimicry and tumour-cell plasticity: Lessons from melanoma. Nat Rev Cancer 2003;3:411-421.

8. Tsutsumi K, Tomomura M, Furuichi T, et al. Palmitoylation-dependent endosomal localization of AATYK1A and its interaction with src. Genes Cells 2008;13:949-964.

9. Tomomura M, Fernandez-Gonzales A, Yano R, et al. Characterization of the apoptosis-associated tyrosine kinase (AATYK) expressed in the CNS. Oncogene 2001;20:1022-1032.

10. Roche S, Fumagalli S, Courtneidge SA. Requirement for src family protein tyrosine kinases in G2 for fibroblast cell division. Science 1995;269:1567-1569.

11. Windham TC, Parikh NU, Siwak DR, et al. Src activation regulates anoikis in human colon tumor cell lines. Oncogene 2002;21: 7797-7807
12. Kilkenny DM, Rocheleau JV, Price J, et al. c-Src regulation of fibroblast growth factor-induced proliferation in murine embryonic fibroblasts. J Biol Chem 2003;278:17448-17454.

13. Biscardi JS, Tice DA, Parsons SJ. c-Src, receptor tyrosine kinases, and human cancer. Adv Cancer Res 1999;76:61-119.

14. Irby RB, Yeatman TJ. Role of src expression and activation in human cancer. Oncogene 2000;19:5636-5642.

15. Summy JM, Gallick GE. Src family kinases in tumor progression and metastasis. Cancer Metastasis Rev 2003;22:337-358.

16. Homsi J, Cubitt C, Daud A. The src signaling pathway: a potential target in melanoma and other malignancies. Expert Opin Ther Targets 2007;11:91-100.

17. Sen B, Johnson FM. Regulation of SRC family kinases in human cancers. J Signal Transduct 2011; 2011: 865819.

18. Homsi J, Cubitt CL, Zhang S, et al. Src activation in melanoma and src inhibitors as therapeutic agents in melanoma. Melanoma Res 2009;19:167-175.

19. Irby RB, Mao W, Coppola D, et al. Activating SRC mutation in a subset of advanced human colon cancers. Nat Genet 1999;21:187-190.

20. Niu G, Bowman $T$, Huang $M$, et al. Roles of activated src and Stat3 signaling in melanoma tumor cell growth. Oncogene 2002;21: 7001-7010.

21. Qi J, Wang J, Romanyuk O, et al. Involvement of src family kinases in $\mathrm{N}$-cadherin phosphorylation and beta-catenin dissociation during transendothelial migration of melanoma cells. Mol Biol Cell 2006;17: 1261-1272.

22. Mirmohammadsadegh A, Hassan M, Bardenheuer W, et al. STAT5 phosphorylation in malignant melanoma is important for survival and is mediated through SRC and JAK1 kinases. J Invest Dermatol 2006;126:2272-2280.

23. Criscuoli ML, Nguyen M, Eliceiri BP. Tumor metastasis but not tumor growth is dependent on src-mediated vascular permeability. Blood 2005;105:1508-1514.

24. Guarino M. Src signaling in cancer invasion. J Cell Physiol 2010;223: 14-26.

25. Kaplan KB, Swedlow JR, Varmus HE, et al. Association of p60c-src with endosomal membranes in mammalian fibroblasts. J Cell Biol 1992;118:321-333.

26. $\mathrm{Lu} \mathrm{Y}, \mathrm{Yu} \mathrm{Q}$, Liu JH, et al. Src family protein-tyrosine kinases alter the function of PTEN to regulate phosphatidylinositol 3-kinase/AKT cascades. J Biol Chem 2003;278:40057-40066.

27. Cantley LC. The phosphoinositide 3-kinase pathway. Science 2002;296:1655-1657.

28. Abram CL, Courtneidge SA. Src family tyrosine kinases and growth factor signaling. Exp Cell Res 2000;254:1-13.

29. Parsons SJ, Parsons JT. Src family kinases, key regulators of signal transduction. Oncogene 2004;23:7906-7909. 